\title{
Vertex-Disjoint Subtournaments of Prescribed Minimum Outdegree or Minimum Semidegree: Proof for Tournaments of a Conjecture of Stiebitz
}

\author{
Nicolas Lichiardopol \\ Nicolas Lichiardopol, Lycée A. de Craponne, 13651 Salonde Provence Cedex, France \\ Correspondence should be addressed to Nicolas Lichiardopol, nicolas.lichiardopol@neuf.fr
}

Received 23 January 2011; Revised 26 April 2011; Accepted 30 May 2011

Academic Editor: Charles Semple

Copyright (C) 2012 Nicolas Lichiardopol. This is an open access article distributed under the Creative Commons Attribution License, which permits unrestricted use, distribution, and reproduction in any medium, provided the original work is properly cited.

It was proved (Bessy et al., 2010) that for $r \geq 1$, a tournament with minimum semidegree at least $2 r-1$ contains at least $r$ vertex-disjoint directed triangles. It was also proved (Lichiardopol, 2010) that for integers $q \geq 3$ and $r \geq 1$, every tournament with minimum semidegree at least $(q-1) r-1$ contains at least $r$ vertex-disjoint directed cycles of length $q$. None information was given on these directed cycles. In this paper, we fill a little this gap. Namely, we prove that for $d \geq 1$ and $r \geq 1$, every tournament of minimum outdegree at least $\left(\left(d^{2}+3 d+2\right) / 2\right) r-\left(d^{2}+d+2\right) / 2$ contains at least $r$ vertex-disjoint strongly connected subtournaments of minimum outdegree $d$. Next, we prove for tournaments a conjecture of Stiebitz stating that for integers $s \geq 1$ and $t \geq 1$, there exists a least number $f(s, t)$ such that every digraph with minimum outdegree at least $f(s, t)$ can be vertex-partitioned into two sets inducing subdigraphs with minimum outdegree at least $s$ and at least $t$, respectively. Similar results related to the semidegree will be given. All these results are consequences of two results concerning the maximum order of a tournament of minimum outdegree $d$ (of minimum semidegree $d$ ) not containing proper subtournaments of minimum outdegree $d$ (of minimum semidegree $d$ ).

\section{Introduction and Definitions}

The definitions which follow are those of [1].

Let $D$ be a digraph. $V(D)$ is the vertex set of $D$ and the order of $D$ is the cardinality of $V(D) . \mathcal{A}(D)$ is the set of the arcs of $T$. Two vertices $x$ and $y$ of $D$ are adjacent, if at least one of the ordered pairs $(x, y)$ and $(y, x)$ is an $\operatorname{arc}$ of $D$. We say that a vertex $y$ is an outneighbor of a vertex $x$ (inneighbour of $x$ ) if $(x, y)(\operatorname{resp} .(y, x))$ is an arc of $D . N_{D}^{+}(x)$ is the set of the outneighbors of $x$ and $N_{D}^{-}(x)$ is the set of the in-neighbors of $x$. The cardinality of $N_{D}^{+}(x)$ is the outdegree $d_{D}^{+}(x)$ of $x$ and the cardinality of $N_{D}^{-}(x)$ is the indegree $d_{D}^{-}(x)$ of $x$. When no confusion is possible, we omit the subscript $D$. We denote by $\delta^{+}(D)$ the minimum outdegree 
of $D$ and by $\delta^{-}(D)$ the minimum indegree of $D$. The minimum semidegree of $D$ is $\delta^{0}(D)=$ $\min \left\{\delta^{+}(D), \delta^{-}(D)\right\}$.

An oriented graph, is a digraph $D$ such that for any two distinct vertices $x$ and $y$ of $D$, at most one of the couples $(x, y)$ and $(y, x)$ is an arc of $D$.

A tournament is an oriented graph $T$ such that any two distinct vertices $x$ and $y$ of $T$ are adjacent. If $A$ and $B$ are subsets of $V(T)$, an arc from $A$ to $B$ is an $\operatorname{arc}(x, y)$ with $x \in A$ and $y \in B$. We denote by $a(A, B)$ the number of the arcs from $A$ to $B$.

It is known and easy to prove that if $n$ is the order of $T$, then $n \geq 2 \delta^{0}(T)+1$ and $\delta^{0}(T) \leq d^{+}(x) \leq n-1-\delta^{0}(T)$ for every vertex $x$ of $T$.

For a subset $S$ of $V(T), T[S]$ is the subtournament induced by the vertices of $S$. For a vertex $x$ of $T, T-x$ is the subtournament induced by the vertices of $T$ distinct from $x$.

For $d \geq 1$, a regular tournament of degree $d$ is a tournament $T$ with $d_{T}^{+}(x)=d_{T}^{-}(x)=d$ for every vertex $x$ of $T$. It is known and easy to prove that the order of $T$ is $2 d+1$.

A path or a cycle of a tournament $T$ always means a directed path or a directed cycle of $T$ and disjoint cycles means vertex-disjoint cycles. A triangle is a directed cycle consisting of three vertices

For distinct vertices $x$ and $y$ of $T$, an $(x, y)$-path is a directed path starting from $x$ and ending at $y$. The tournament $T$ is said to be strongly connected, or briefly strong, if for any distinct vertices $x$ and $y$, there exists an $(x, y)$-path. It is well known (Camion Theorem) that a tournament $T$ is strong if and only if it contains a Hamiltonian cycle. The strong connectivity of $T$ is the smallest nonnegative integer $k(T)$ such that there exists a subset of $k(T)$ vertices of $V(T)$ disconnecting $T$. For $k \geq 1$, a $k$-strong tournament is a tournament of strong connectivity at least $k$.

If $V_{1}$ and $V_{2}$ are two vertex-disjoint subsets of $V(T)$, we say that $V_{1}$ dominates $V_{2}$, if for every pair $\{x, y\}$ with $x \in V_{1}$ and $y \in V_{2},(x, y)$ is an arc of $T$ (which means that there is no arc from $V_{2}$ to $V_{1}$ ). If $T_{1}$ and $T_{2}$ are two tournaments with disjoint vertex sets, $T_{1} \rightarrow T_{2}$ is the tournament whose vertex set is $V\left(T_{1}\right) \cup V\left(T_{2}\right)$ and whose arcs are those of $T_{1}$ and $T_{2}$ and the ordered pairs $(x, y)$ with $x \in V\left(T_{1}\right)$ and $y \in V\left(T_{2}\right)$. It is known and easy to prove that a tournament $T$ is nonstrong if and only if there exists a partition $A, B$ of $V(T)$ such that $T=T[A] \rightarrow T[B]$.

A minimum outdegree minimal tournament is a tournament $T$ such that every proper subtournament of $T$ has minimum outdegree at most $\delta^{+}(T)-1$. If $\delta^{+}(T)=d$, we say that $T$ is a minimum outdegree $d$ minimal tournament. Similarly, one can define the notion of minimum indegree minimal tournament. A minimum semidegree minimal tournament is a tournament $T$ such that every proper subtournament of $T$ has minimum semidegree at most $\delta^{0}(T)-1$. If $\delta^{0}(T)=d$, we say that $T$ is a minimum semidegree $d$ minimal tournament.

In a recent paper, Bessy et al. (see [2]) proved that for $r \geq 1$, a tournament with minimum outdegree and minimum indegree both greater or equal to $2 r-1$ contains at least $r$ vertex-disjoint directed triangles. In a more recent paper (see [3]), the author generalized this result, by proving that for given integers $q \geq 3$ and $r \geq 1$, every tournament with minimum outdegree and minimum indegree both greater or equal to $(q-1) r-1$ contains at least $r$ vertexdisjoint directed cycles of length $q$. None information was given on these directed cycles. In this paper, we fill a little this gap. More precisely, we prove:

Theorem 1.1. For $d \geq 2$ and for $r \geq 1$, every tournament $T$ of minimum outdegree at least $\left(\left(d^{2}+\right.\right.$ $3 d+2) / 2) r-\left(d^{2}+d+2\right) / 2$ contains at least $r$ vertex-disjoint strong subtournaments of minimum outdegree $d$. 
Concerning the minimum semidegree, we prove the following.

Theorem 1.2. For $d \geq 2$ and for $r \geq 1$, every tournament $T$ of minimum semidegree at least $\left(d^{2}+\right.$ $3 d+2) r-d^{2}-2 d-2$ contains at least $r$ vertex-disjoint subtournaments of minimum semidegree $d$.

In [4], Stiebitz proposed the following conjecture.

Conjecture 1. For given integers $s \geq 1$ and $t \geq 1$, there exists a least number $f(s, t)$ such that the vertices of any simple digraph with minimum outdegree at least $f(s, t)$ can be partitioned into two sets inducing subdigraphs with minimum outdegree at least $s$ and at least $t$, respectively.

A natural generalization given by Alon (Problem 1 in [5]) is as follows.

Conjecture 2. For an integer $k \geq 2$ and for given positive integers $d_{1}, \ldots d_{k}$, there exists a least number $F\left(d_{1}, \ldots d_{k}\right)$ such that the vertices of any simple digraph $D$ with minimum outdegree at least $F\left(d_{1}, \ldots d_{k}\right)$ can be partitioned into $k$ sets $V_{1}, \ldots V_{k}$ such that $\delta^{+}\left(D\left[V_{i}\right]\right) \geq d_{i}$ for $1 \leq i \leq$ $k$.

It is easy to prove by induction that the existence of $f(s, t)$ implies the existence of $F\left(d_{1}, \ldots d_{k}\right)$. In this paper, we prove that Stiebitz's conjecture is true for tournaments (with a supplementary constraint), namely, we prove the following.

Theorem 1.3. For given integers $s \geq 1$ and $t \geq 1$, the vertices of any tournament $T$ with minimum outdegree at least $\left(s^{2}+3 s+2\right) / 2+t$ can be partitioned into two sets inducing subtournaments $T_{1}$ and $T_{2}$ with $T_{1}$ strongly connected and of minimum outdegree at least $s$ and with $T_{2}$ of minimum outdegree at least $t$.

This result will allow us to prove, for tournaments, the generalized conjecture of Stiebitz. Relatively to the minimum semidegree, we state the following.

Theorem 1.4. For given integers $s \geq 1$ and $t \geq 1$, the vertices of any tournament $T$ with minimum semidegree at least $s^{2}+3 s+2+t$ can be partitioned into two sets inducing subtournaments $T_{1}$ and $T_{2}$ with $T_{1}$ of minimum semidegree at least $s$ and with $T_{2}$ of minimum semidegree at least $t$.

Here also, a generalization is possible.

\section{Results on Minimum Outdegree and Minimum Semidegree Minimal Tournaments}

We begin with the following theorem,

Theorem 2.1. For $d \geq 1$, any minimum outdegree d minimal tournament, is strong.

Proof. Suppose the opposite. Then there exists a partition $(A, B)$ of $V(T)$ such that $A$ dominates $B$. Then $T[B]$ is a proper subtournament of $T$ of minimum outdegree at least $d$, which is impossible.

We continue with the following,

Theorem 2.2. For $d \geq 1$, any tournament of minimum outdegree at least $d$ contains a minimum outdegree d minimal subtournament. 


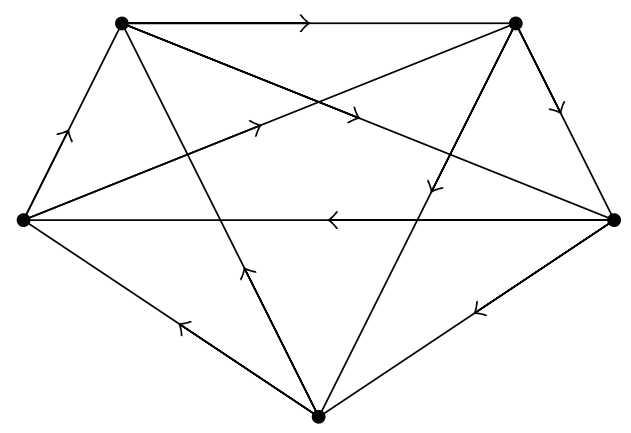

Figure 1: Minimum outdegree 2 minimal tournament $T_{1}$.

Proof. Let $s$ be the smallest order of the subtournaments of $T$ having minimum outdegree at least $d$. There exists a subtournament $T^{\prime}$ of $T$ of order $s$ and of minimum outdegree at least $d$. Clearly, $T^{\prime}$ is a minimum outdegree $d$ minimal subtournament. following,

Concerning the order of a minimum outdegree $d$ minimal tournament, we state the

Theorem 2.3. For $d \geq 1$, if $T$ is a minimum outdegree $d$ minimal tournament of order $n$, one has $n \leq\left(d^{2}+3 d+2\right) / 2$.

Proof. Let $M$ be the set of the vertices of $T$ of outdegree $d$ and let $m$ be its cardinality. For every vertex $x$ of $T$, the tournament $T-x$ has minimum outdegree $d-1$, and this means that $x$ has at least one in-neighbor in $M$. Then, the number of the arcs from $M$ to $V(T) \backslash M$ is at least $n-m$. On the other hand, the number of the arcs from $M$ to $V(T) \backslash M$ is exactly $m d-(m(m-1)) / 2$. It follows $m d-(m(m-1)) / 2 \geq n-m$, hence $m^{2}-(2 d+3) m+2 n \leq 0$. This implies $(2 d+3)^{2}-8 n \geq 0$, hence $n \leq(2 d+3)^{2} / 8$ and since $n$ is an integer, we get $n \leq\left((2 d+3)^{2}-1\right) / 8$, that is $n \leq\left(d^{2}+3 d+2\right) / 2$.

We note that $\left(2 d+3-\sqrt{(2 d+3)^{2}-8 n}\right) / 2 \leq m \leq\left(2 d+3-\sqrt{(2 d+3)^{2}-8 n}\right) / 2$. When $d=2$, we get $n \leq 6$. It is easy to prove that there are four minimum outdegree 2 minimal tournaments: the regular tournament $T_{1}$ of order 5 (Figure 1), and three nonisomorphic tournaments $T_{2}, T_{3}$, and $T_{4}$ of order 6 (Figure 2). The outdegree sequence of $T_{2}$ is $(2,2,2,3,3,3)$, and the outdegree sequence of the tournaments $T_{3}$ and $T_{4}$ is $(2,2,2,2,3,4)$. We observe that $T_{3}$ and $T_{4}$ are not 2-connected (the two others yes).

We claim that for every integer $d \geq 1$ the bound of Theorem 2.3 is reached. To be more precise, we claim that for every integer $d \geq 1$, there exists a minimum outdegree $d$ minimal tournament of order $\left(d^{2}+3 d+2\right) / 2$ and of strong connectivity 1 . We are going to prove this, by induction on $d$. Clearly, the assertion is true for $d=1$. Suppose that the assertion is true up to the row $d-1, d \geq 2$. Let then $T_{d-1}$ be a minimum outdegree $d-1$ minimal tournament of order $\left((d-1)^{2}+3(d-1)+2\right) / 2=\left(d^{2}+d\right) / 2$. Let $T_{d}$ be the tournament defined as follows.

(i) The vertices of $T_{d}$ are the vertices of $T_{d-1}$ and $d+1$ additional vertices $x$ and $y_{1}, y_{2}, \ldots, y_{d}$.

(ii) The $\operatorname{arcs}$ of $T_{d}$ are the $\operatorname{arcs}$ of $T_{d-1}$, the couples $\left(x, y_{i}\right), 1 \leq i \leq d$, the couples $(z, x)$ with $z \in V\left(T_{d-1}\right)$, the couples $\left(y_{i}, z\right)$ with $1 \leq i \leq d$ and $z \in V\left(T_{d-1}\right)$ and the arcs of an arbitrary tournament with $A=\left\{y_{1}, \ldots, y_{d}\right\}$ as vertex set. It is easy to see that 

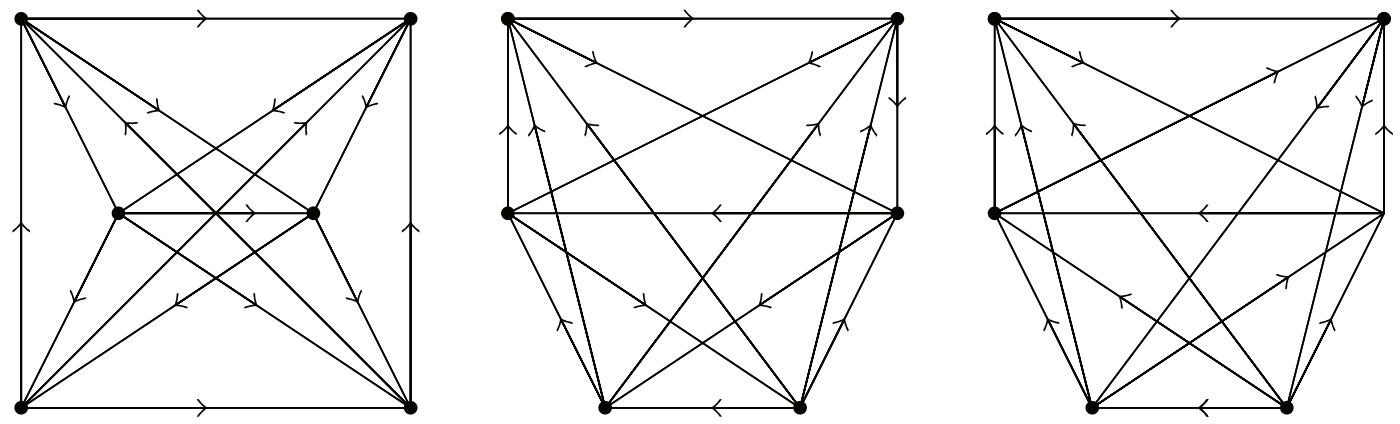

Figure 2: Minimum outdegree 2 minimal tournaments $T_{2}, T_{3}$, and $T_{4}$.

$T_{d}$ is a minimum outdegree $d$ minimal tournament of order $\left(d^{2}+d\right) / 2+d+1=$ $\left(d^{2}+3 d+2\right) / 2$ and of strong connectivity 1 (because $x$ disconnects $\left.T_{d}\right)$. So the assertion is true for $d$ and consequently the result is proved.

In fact, there are minimum outdegree $d$ minimal tournaments of strong connectivity 1 of order smaller than $\left(d^{2}+3 d+2\right) / 2$. Indeed, if we take a regular tournament of degree $d-1$, with the above construction, we get a minimum outdegree $d$ minimal tournament of connectivity 1 and of order $2(d-1)+1+d+1=3 d$. We think that $3 d$ is the minimum order of a minimum outdegree $d$ minimal tournament of connectivity 1 .

By minimum outdegree $d$ critical tournament, we mean a tournament $T$ of minimum outdegree $d$ such that for every vertex $x$ of $T$, the tournament $T-x$ has minimum out degree $d-1$. It is clear that a minimum outdegree $d$ minimal tournament is minimum outdegree critical. By the way, we observe that in the proof of Theorem 2.3, we use only the fact that $T$ is minimum outdegree $d$ critical and as the obtained upper bound is reached, another proof using the fact that $T$ is minimum outdegree $d$ minimal cannot improve this upper bound. However, we claim that for $d \geq 3$, the notion of minimum outdegree $d$ minimal tournament does not coincide with the notion of minimum outdegree $d$ critical tournament. Indeed, let $T^{\prime}$ be a regular tournament of degree $d$ and consider a vertex $x$ of $T^{\prime}$. We define the tournament $T$ in the following way.

(i) The vertices of $T$ are the vertices of $T^{\prime}$ and the additional vertices $a, b$ and $c$ of a directed triangle $\Delta$.

(ii) $V(\Delta)$ is dominated by $N_{T^{\prime}}^{+}(x), x$ is dominated by $V(\Delta)$. To each vertex of $V(\Delta)$ we join exactly $d-2$ outneighbors in $N_{T^{\prime}}^{-}(x)$ so that every vertex of $N_{T^{\prime}}^{-}(x)$ has at least one in-neighbor in $V(\Delta)$ (this is possible when $d \geq 3$ ).

$T$ has minimum outdegree $d$ and the vertices $x, a, b$, and $c$ are of outdegree $d$. It is easy to see that every vertex of $T$ is dominated by a vertex of $\{x, a, b, c\}$. This means that $T$ is minimum outdegree $d$ critical. Manifestly, since $T^{\prime}$ is a proper subtournament of $T$ of minimum outdegree $d, T$ is not minimum outdegree $d$ minimal. This corroborates our statement. However, as for minimum outdegree minimal tournaments, we have the following.

Theorem 2.4. For $d \geq 1$, any critically outdegree $d$ tournament $T$ is strong.

Proof. Suppose the opposite. Then there exists a partition $(A, B)$ of $V(T)$ such that $A$ dominates $B$. Then $T[B]$ is a tournament of minimum outdegree at least $d$, which implies 
$|B| \geq 2 d+1>d$. Consider a vertex $x$ of $A$. An in-neighbor of $x$ is in $A$ and then its outdegree is greater than $d$. This means that $T-x$ is a tournament of minimum outdegree $d$, which is not possible. Consequently $T$ is strong.

For minimum semidegree minimal tournaments, the situation is a little different. Already, we observe that a minimum semidegree minimal tournament is not necessarily strong. Indeed, if $T_{1}$ is a minimum indegree $d$ minimal tournament and if $T_{2}$ is a minimum outdegree $d$ minimal tournament, vertex disjoint with $T_{1}$, it is easy to prove that $T_{1} \rightarrow T_{2}$ is a nonstrong minimum semidegree $d$ minimal tournament. As for Theorem 2.2, we have the following.

Theorem 2.5. For $d \geq 1$, any tournament of minimum semidegree at least $d$ contains a minimum semidegree d minimal subtournament.

Proof. Let $s$ be the smallest order of the subtournaments of $T$ having minimum semidegree at least $d$. There exists a subtournament $T^{\prime}$ of $T$ of order $s$ and of minimum semidegree at least $d$. Clearly, $T^{\prime}$ is a minimum semidegree $d$ minimal subtournament.

As regards the maximum order, we state the following.

Theorem 2.6. For $d \geq 2$, if $T$ is a minimum semidegree $d$ minimal tournament of order $n$ and of minimum semidegree $d$, we have $n \leq d^{2}+3 d+2$.

Proof. We have $n \geq 2 d+1$ and if $n=2 d+1$, the theorem is proved. So, we may suppose $n>2 d+1$. For every vertex $x$ of $T$, the tournament $T-x$ has minimum semidegree $d-1$. This means that $x$ has an in-neighbor of outdegree $d$ or an outneighbor of indegree $d$ (here "or" is not exclusive). Denote by $M_{1}$ the set of the vertices of $T$ of outdegree $d$ and by $M_{2}$ the set of the vertices of $T$ of indegree $d$. Since $n>2 d+1, M_{1}$ and $M_{2}$ are disjoint. Let $m_{1}$ and $m_{2}$ be the cardinalities of the sets $M_{1}$ and $M_{2}$. If $m_{1}=0$ or if $m_{2}=0$, as in the proof of Theorem 2.3, we get $n \leq\left(d^{2}+3 d+2\right) / 2$ and then the theorem is proved. So, we may suppose $m_{1} \geq 1$ and $m_{2} \geq 1$. Denote by $M_{3}$ the set of the vertices of $T$ not in $M_{1} \cup M_{2}$ and having at least one in-neighbor in $M_{1}$, and by $m_{3}$ the cardinality of $M_{3}$. Let $M_{4}=V(T) \backslash\left(M_{1} \cup M_{2} \cup M_{3}\right)$ and $m_{4}=\left|M_{4}\right|$. Every possible vertex of $M_{4}$ has a least one outneighbor in $M_{2}$.

We have $a\left(M_{1}, M_{3}\right) \geq m_{3}$, and since $a\left(M_{1}, M_{3}\right)=m_{1} d-\left(m_{1}\left(m_{1}-1\right)\right) / 2-a\left(M_{1}, M_{2}\right)-$ $a\left(M_{1}, M_{4}\right)$, it follows that

$$
m_{1} d-\frac{m_{1}\left(m_{1}-1\right)}{2}-a\left(M_{1}, M_{2}\right)-a\left(M_{1}, M_{4}\right) \geq m_{3}
$$

Similarly, we get

$$
m_{2} d-\frac{m_{2}\left(m_{2}-1\right)}{2}-a\left(M_{1}, M_{2}\right)-a\left(M_{3}, M_{2}\right) \geq m_{4}
$$

From (2.1) and (2.2), we get by addition $\left(m_{1}+m_{2}\right) d+\left(m_{1}+m_{2}\right) / 2-\left(m_{1}^{2}+m_{2}^{2}\right) / 2-2 a\left(M_{1}, M_{2}\right)-$ $a\left(M_{1}, M_{4}\right)-a\left(M_{3}, M_{2}\right) \geq n-m_{1}-m_{2}$. It is easy to prove that $m_{1}^{2}+m_{2}^{2} \geq\left(m_{1}+m_{2}\right)^{2} / 2$. It follows $\left(m_{1}+m_{2}\right)(d+(1 / 2))-\left(m_{1}+m_{2}\right)^{2} / 4-2 a\left(M_{1}, M_{2}\right)-a\left(M_{1}, M_{4}\right)-a\left(M_{3}, M_{2}\right) \geq$ $n-\left(m_{1}+m_{2}\right)$, hence $\left(m_{1}+m_{2}\right)(d+(1 / 2))-\left(m_{1}+m_{2}\right)^{2} / 4 \geq n-\left(m_{1}+m_{2}\right)$. This yields 
$\left(m_{1}+m_{2}\right)^{2}-(4 d+6)\left(m_{1}+m_{2}\right)+4 n \leq 0$. This implies $(2 d+3)^{2}-4 n \geq 0$, hence $n \leq(2 d+3)^{2} / 4$ and since $n$ is an integer, we get $n \leq\left((2 d+3)^{2}-1\right) / 4$, that is $n \leq d^{2}+3 d+2$.

Concerning the number $m_{1}+m_{2}$ of the vertices $x$ of $T$ with either outdegree $d$ or indegree $d$, we have $2 d+3-\sqrt{(2 d+3)^{2}-4 n} \leq m_{1}+m_{2} \leq 2 d+3-\sqrt{(2 d+3)^{2}-4 n}$. When $d=2$, we get $n \leq 12$, and this bound is reached. Indeed if $T_{1}$ is a critically indegree $d$ tournament of order 6 and if $T_{2}$ is a a critically outdegree $d$ tournament of order 6 , then $T_{1} \rightarrow T_{2}$ is a critically semidegree $d$ tournament of order 6 .

\section{Proofs of Theorems 1.1 and 1.2}

\subsection{Proof of Theorem 1.1}

Since $\delta^{+}(T) \geq d$, by Theorem 2.2, T contains minimum outdegree $d$ minimal subtournaments. Let $s>0$ be the maximum number of vertex-disjoint minimum outdegree $d$ minimal subtournaments. Then there exist $s$ vertex-disjoint minimum outdegree $d$ minimal subtournaments $T_{1}, \ldots T_{S}$, and by Theorem 2.3 , these tournaments cover a set $S$ with at most $\left(\left(d^{2}+3 d+2\right) / 2\right) S$ vertices of $T$. Suppose that $s<r$. A vertex $x$ of $V(T) \backslash S$ has at most $\left(\left(d^{2}+3 d+2\right) / 2\right) s$ outneighbors in $S$. It follows that $x$ has at least $\left(\left(d^{2}+3 d+2\right) / 2\right) r-\left(d^{2}+d+2\right) / 2-\left(\left(d^{2}+3 d+\right.\right.$ $2) / 2) s \geq d$ outneighbors in $V(T) \backslash S$. This means that the subtournament induced by $V(T) \backslash S$ has minimum outdegree at least $d$ and then by Theorem 2.2, it contains a minimum outdegree $d$ minimal subtournament. But by maximality of $s$, this is not possible. Consequently $s \geq r$ and therefore $T$ contains $r$ minimum outdegree $d$ minimal subtournaments. Since these tournaments are strong (Theorem 2.1), the result is proved.

\subsection{Proof of Theorem 1.2}

Since $\delta^{0}(T) \geq d$, by Theorem 2.4, $T$ contains minimum semidegree $d$ minimal subtournaments. Let $s>0$ be the maximum number of vertex-disjoint minimum semidegree $d$ minimal subtournaments. Then there exist $s$ vertex-disjoint minimum semidegree $d$ minimal subtournaments, and by Theorem 2.5, these tournaments cover a set $S$ with at most $\left(d^{2}+3 d+\right.$ $2) s$ vertices of $T$. Suppose that $s<r$. A vertex $x$ of $V(T) \backslash S$ has at most $\left(d^{2}+3 d+2\right) s$ outneighbors in $S$ and at most $\left(d^{2}+3 d+2\right) s$ in-neighbors in $S$. It follows that $x$ has at least $\left(d^{2}+3 d+2\right) r-d^{2}-2 d-2-\left(d^{2}+3 d+2\right) s \geq d$ outneighbors in $V(T) \backslash S$ and at least $\left(d^{2}+3 d+2\right) r-d^{2}-2 d-2-\left(d^{2}+3 d+2\right) s \geq d$ in-neighbors in $V(T) \backslash S$. This means that the subtournament induced by $V(T) \backslash S$ has minimum semidegree at least $d$ and then by Theorem 2.4, it contains a minimum semidegree $d$ minimal subtournament. But by maximality of $s$, this is not possible. Consequently $s \geq r$ and therefore $T$ contains $r$ minimum semidegree $d$ minimal subtournaments, which proves the result.

\section{Proofs of Theorems 1.3 and 1.4 and Generalizations}

Since the proof of Theorem 1.4 is similar to that of Theorem 1.3, we prove only Theorem 1.3.

By Theorem 2.2, $T$ contains a minimum outdegree $s$ minimal subtournament $T_{1}$, which is strong by Theorem 2.1. By Theorem 2.3, the order of $T_{1}$ is at most $\left(s^{2}+3 s+2\right) / 2$. Let $T_{2}$ be the subtournament induced by $V(T) \backslash V\left(T_{1}\right)$. A vertex $x$ of $T_{2}$ has at least $\left(s^{2}+3 s+2\right) / 2+t$ outneighbors in $V(T)$ and at most $\left(s^{2}+3 s+2\right) / 2$ outneighbors in $V\left(T_{1}\right)$. It follows that $x$ has at least $t$ outneighbors in $V\left(T_{2}\right)$. This means that $T_{2}$ has minimum outdegree at least $t$, and consequently the theorem is proved. 
This being established, an easy induction, gives the following.

Theorem 4.1. For an integer $k \geq 2$ and for given positive integers $d_{1}, \ldots d_{k}$, the vertices of any tournament $T$ with minimum outdegree at least $\left(d_{1}^{2}+3 d_{1}+2\right) / 2+\cdots+\left(d_{k-1}^{2}+3 d_{k-1}+2\right) / 2+d_{k}$, can be partitioned into $k$ sets $V_{1}, \ldots, V_{k}$ so that $\delta^{+}\left(T\left[V_{i}\right]\right) \geq d_{i}$ for $1 \leq i \leq k$ and $T\left[V_{i}\right]$ is strong for $1 \leq i \leq k-1$.

Proof. The assertion is true for $k=2$ because it is Theorem 1.3. Suppose that the assertion is true up to the row $k-1, k \geq 3$ and let us study for $k$. So, let $T$ be a tournament with minimum outdegree at least $\left(d_{1}^{2}+3 d_{1}+2\right) / 2+\cdots+\left(d_{k-1}^{2}+3 d_{k-1}+2\right) / 2+d_{k}$. By Theorem 1.3, V(T) can be partitioned into two sets $V_{1}$ and $V^{\prime}$, so that $T\left[V_{1}\right]$ is strong, of minimum outdegree at least $d_{1}$ and $T^{\prime}=T\left[V^{\prime}\right]$ is of minimum out degree at least $\left(d_{2}^{2}+3 d_{2}+2\right) / 2+\cdots+\left(d_{k-1}^{2}+3 d_{k-1}+2\right) / 2+d_{k}$. By induction hypothesis $V\left(T^{\prime}\right)=V^{\prime}$ can be partitioned into $k-1$ sets $V_{2}, \ldots, V_{k}$ so that $T^{\prime}\left[V_{i}\right]=$ $T\left[V_{i}\right]$ is of minimum outdegree at least $d_{i}$ for $2 \leq i \leq k$ and $T^{\prime}\left[V_{i}\right]=T\left[V_{i}\right]$ is strong for $2 \leq i \leq k-1$. It follows, by considering also $V_{1}$, that the assertion is true for $k$ and therefore the result is proved.

In fact, we are able to prove Conjecture 1 (and then Conjecture 2) for a larger class of oriented graphs (including tournaments). Namely, we state the following.

Theorem 4.2. For given integers $s \geq 1, t \geq 1$ and $r \geq 0$, let $D$ be an oriented graph with minimum outdegree at least $\left((s+r)^{2}+3(s+r)+2\right) / 2+t+r$ and such that for every vertex $x$ there exist at most $r$ vertices of $D$ nonadjacent with $x$. Then the vertices of $D$ can be partitioned into two sets inducing oriented graphs $D_{1}$ and $D_{2}$ with $D_{1}$ of minimum outdegree at least $s$, and strongly connected when $r=0$, and with $D_{2}$ of minimum outdegree at least $t$.

Proof. When $r=0, D$ is a tournament and then the result is proved. Therefore we may suppose $r \geq 1$. By orienting every nonedge of $D$, we obtain a tournament $T$ having $D$ as spanning subdigraph. Since $T$ has minimum out degree at least $\left((s+r)^{2}+3(s+r)+2\right) / 2+t+r$, by Theorem 1.3, $V(T)=V(D)$ can be partitioned into two sets inducing subtournaments $T_{1}$ and $T_{2}$ with $T_{1}$ of minimum outdegree at least $s+r$ and with $T_{2}$ of minimum outdegree at least $t+r$. Since every vertex of $V(D)$ has at most $r$ outneighbors in $T$ which are not outneighbors in $D$, by deleting all the arcs of $T_{1}$ and $T_{2}$ which are not arcs of $D$, we get a spanning subdigraph $D_{1}$ of $T_{1}$, induced in $D$ by $V\left(T_{1}\right)=V\left(D_{1}\right)$, with minimum outdegree at least $s$ and a spanning subdigraph $D_{2}$ of $T_{2}$, induced in $D$ by $V\left(T_{2}\right)=V\left(D_{2}\right)$ and with minimum outdegree at least $t$. Since $V\left(D_{1}\right), V\left(D_{2}\right)$ is a partition of $V(D)$, the theorem is proved.

For example an oriented graph $D$ of minimum outdegree at least 34 such that every vertex is nonadjacent with at most 4 vertices is vertexdecomposable into two oriented graphs of minimum out degree 2 .

The proof of Theorem 1.4 (related to the semidegree) is similar to that of Theorem 1.3 and here also a generalization is possible.

\section{An Open Problem of Thomassen}

In [6], Reid proposed the following problem raised by Thomassen.

Problem 1. Let $r$ and $q$ be positive integers, does there exists a positive integer $s=s(r, q)$ so that all but a finite number of s-strong tournaments can be vertex-partitioned into an $r$-strong and a $q$-strong subtournament? 
By minimal $k$-strong tournament, we mean a $k$-strong tournament $T$ such that every proper subtournament of $T$ has strongconnectivity at most $k-1$. By critical $k$ strong tournament, we mean a $k$-strong tournament $T$ such that for every vertex $x$, the subtournament $T-x$ has strong connectivity at most $k-1$. We think it is as follows.

Conjecture 3. For a given integer $k>0$, there exists a function $f(k)$ such that every minimal $k$-strong tournament is of order at most $f(k)$.

It is easy to prove that a positive answer to this conjecture would give a positive answer to Thomassen's open problem. It is known that the conjecture is false, when we replace minimal $k$-strong tournament by critical $k$-strong tournament, but in spite of that, we maintain our conjecture.

\section{Acknowledgment}

Many thanks are due to the referees for their helpful comments.

\section{References}

[1] J. Bang-Jensen and G. Gutin, Digraphs, Springer Monographs in Mathematics, Springer, New York, NY, USA, 2001.

[2] S. Bessy, N. Lichiardopol, and J.-S. Sereni, "Two proofs of the Bermond-Thomassen conjecture for tournaments with bounded minimum in-degree," Discrete Mathematics, vol. 310, no. 3, pp. 557-560, 2010.

[3] N. Lichiardopol, "Vertex-disjoint directed cycles of prescribed length in tournaments with given minimum out-degree and in-degree," Discrete Mathematics, vol. 310, no. 19, pp. 2567-2570, 2010.

[4] M. Stiebitz, "Decompositions of graphs and digraphs," KAM Series in Discrete MathematicsCombinatorics-Operation Research 95-309, 56-59.

[5] N. Alon, "Splitting digraphs," Combinatorics, Probability and Computing, vol. 15, no. 6, pp. 933-937, 2006.

[6] K. B. Reid, "Two complementary circuits in two-connected tournaments," in Cycles in Graphs (Burnaby, B.C., 1982), vol. 115 of North-Holland Math. Stud., pp. 321-334, North-Holland, Amsterdam, The Netherlands, 1985. 


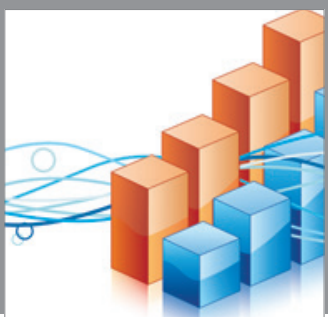

Advances in

Operations Research

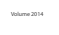

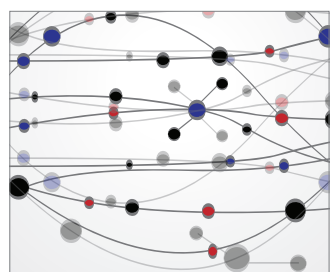

\section{The Scientific} World Journal
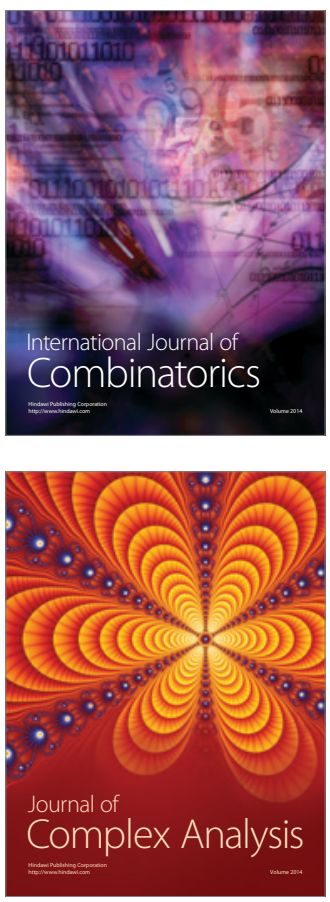

International Journal of

Mathematics and

Mathematical

Sciences
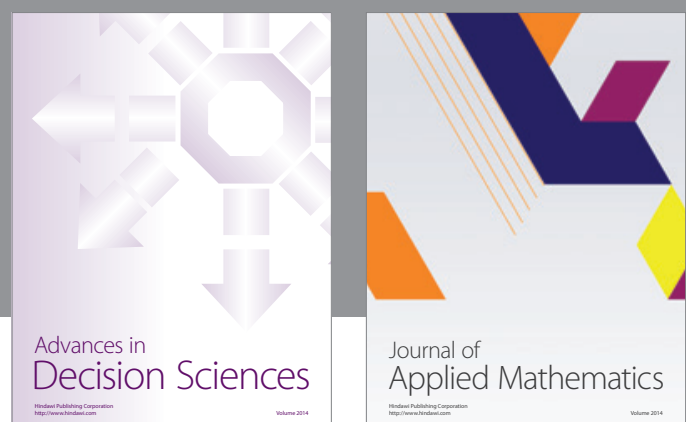

Journal of

Applied Mathematics
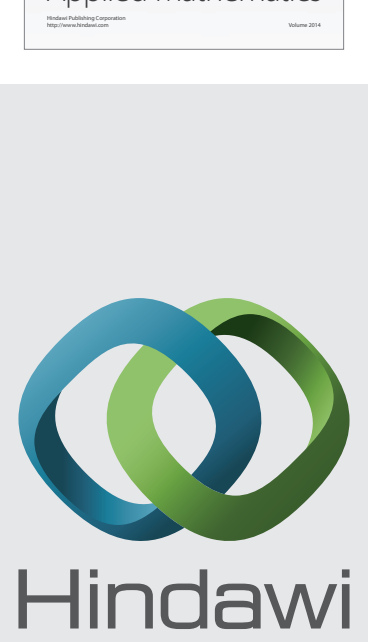

Submit your manuscripts at http://www.hindawi.com
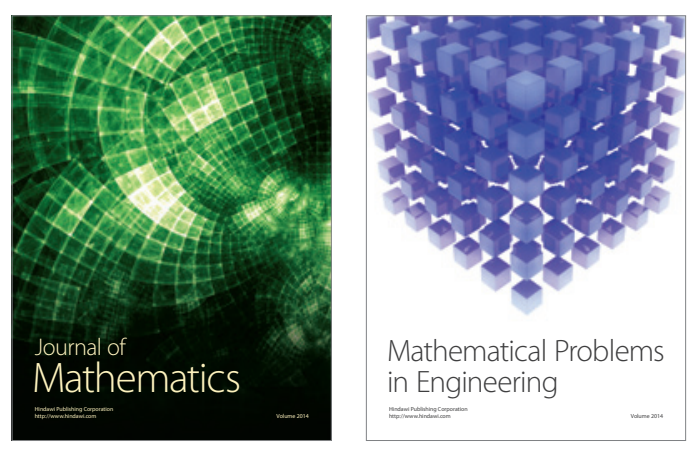

Mathematical Problems in Engineering
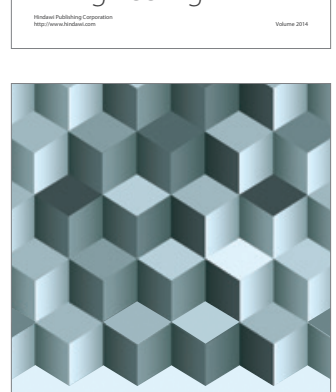

Journal of

Function Spaces
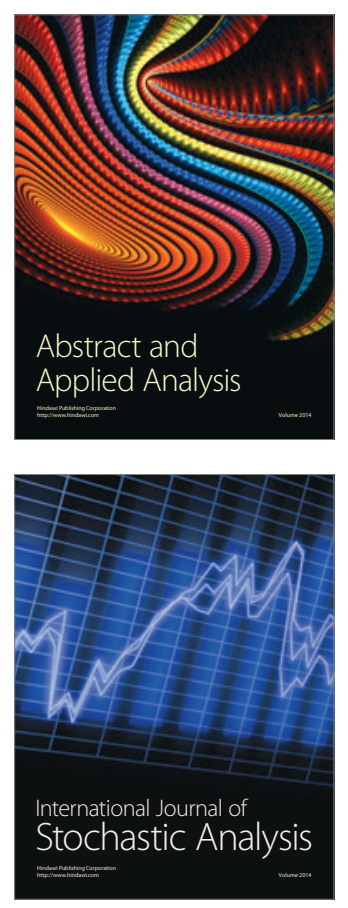

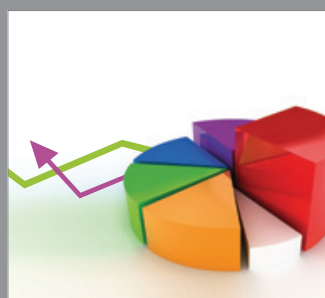

ournal of

Probability and Statistics

Promensencen
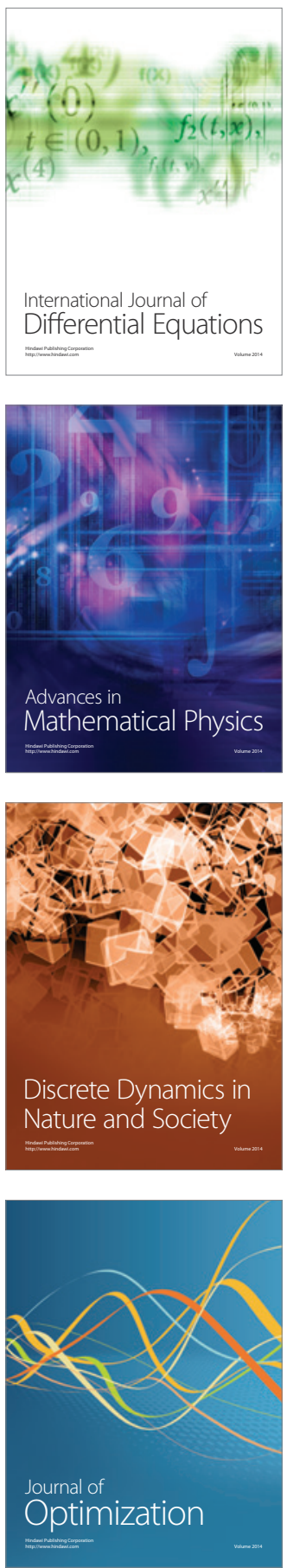